\title{
Spontaneous bilateral tubal ectopic pregnancy
}

\author{
Jeevan P Marasinghe ${ }^{1}$, George Condous ${ }^{2}$ and W I Amarasinghe ${ }^{3}$ \\ (Index words: tubal ectopic pregnancy, transvaginal ultrasound, expectant management, hCG, laparotomy)
}

\begin{abstract}
A 28-year old woman presented at eight weeks and four days of gestation, according to her menstrual dates, complaining of painless vaginal bleeding for three days. Her urinary pregnancy test was positive. Initial transvaginal ultrasound demonstrated an irregular complex structure with a fluid filled centre in the right adnexum. Despite the diagnosis of a possible underlying unruptured right tubal ectopic pregnancy, she declined surgical intervention and was managed expectantly as an inpatient. When she complained of increasing abdominal pain with haemodynamic instability, an emergency laparotomy was performed and a diagnosis of bilateral tubal ectopic pregnancy was made.
\end{abstract}

\section{Case report}

A 28-year old nulliparous married woman presented with painless vaginal bleeding after a period of amenorrhoea of eight weeks and four days. Her urinary pregnancy test was positive. She had no risk factors for ectopic pregnancy (EP). Her clinical examination was unremarkable and she was haemodynamically stable. A transvaginal ultrasound scan (TVS) showed an empty uterus with an endometrial thickness of $12 \mathrm{~mm}$ with decidual changes. There was an irregular complex structure with a fluid filled centre in the right adnexum measuring $29 \times 32 \mathrm{~mm}$. This structure demonstrated peripheral hypervacularization with colour Doppler imaging. The left adnexum was normal. An abnormal rise in quantitative serum human chorionic gonadotrophin (hCG) $(22,996 \mathrm{IU} / \mathrm{L}$ on admission to $25,432 \mathrm{IU} / \mathrm{L}$ ) was noted at 48 hours; hCG ratio (hCG $48 \mathrm{hr} / \mathrm{hCG} 0 \mathrm{hr}) 1.11$.

The possibility of an underlying unruptured right tubal EP was conveyed to the couple. They declined surgical intervention and insisted on expectant management. The patient was clearly warned about the possibility of subsequent tubal rupture and emergency surgical intervention, and was kept under careful observation as an inpatient. Two days after admission she complained of increasing lower abdominal pain and there was clinical and ultrasonic evidence of haemoperitoneum. An emergency laparotomy was performed and this showed bilateral tubal EPs with $600 \mathrm{ml}$ of blood in the peritoneal cavity. Left linear salpingotomy and right salpingectomy were performed. Histology confirmed the presence of chorionic villi in both specimens.

\section{Discussion}

The rates of EP are increasing in industrialized countries due to increasing prevalence of chlamydia and the growing use of assisted reproductive techniques in an ever ageing maternal population [1]. Concurrently, maternal morbidity and mortality associated with EPs are falling [2]. However, spontaneous bilateral EP is a very rare condition accounting for 1 in 750 to 1 in 1580 of EPs [3]. Screening for bilateral EP in low-risk women is not justifiable. However, bilateral EP can occur following invitro fertilization and multiple embryo transfers (IVF-ET) [4]. Therefore careful assessment of both adnexae using TVS is advisable in pregnancies following assisted reproductive technologies. The cost effectiveness of such surveillance remains dubious, and pre-operative diagnosis of bilateral EP remains a real clinical challenge for gynaecologists and Early Pregnancy Units (EPUs).

In our case, unruptured bilateral EPs were not diagnosed until surgical exploration. One of the shortcomings in the management of this woman was the late surgical intervention. Although the possibility of an unruptured right tubal ectopic pregnancy was considered after initial TVS, bilateral tubal pathology was not noted. Had the correct diagnosis been made at the initial scan, the recommendation for laparoscopic intervention would have been much stronger. Notwithstanding the fact that a pre-operative ultrasound diagnosis was not made, the index of suspicion should have been much higher due to the unusually high serum hCG levels at presentation combined with the hCG ratio of 1.11 after 48 hours [5]. Most EPs should be diagnosed pre-operatively using grey scale 2D TVS, and importantly, does not require the use of colour Doppler [6].

${ }^{1}$ De Soyza Hospital for Women, Colombo, Sri Lanka, ${ }^{2}$ Early Pregnancy and Advanced Endosurgery Unit, Nepean Clinical School, University of Sydney, Australia and ${ }^{3}$ Department of Obstetrics and Gynaecology, Faculty of Medicine, University of Peradeniya, Sri Lanka.

Correspondence: JPM, e-mail: <jeevanmarasinghe@yahoo.com>. Received 24 April 2008 and revised version accepted 8 November 2008. Competing interests: none declared. 


\section{References}

1. Murray H, Baakdah H, Bardell T, Tulandi T. Diagnosis and treatment of ectopic pregnancy. Canadian Medical Association Journal 2005; 173: 905-12.

2. Lewis $G$ (ed). The confidential inquiry into maternal and child health (CEMACH). Saving mothers' lives: reviewing maternal deaths to make motherhood safer 2003-2005. The seventh report on confidential enquiries into maternal deaths in the United Kingdom. London: CEMACH, 2007.

3. Adair CD, Benrubi GI, Sanchez-Ramos L, Rhatigan R. Bilateral tubal ectopic pregnancies after bilateral partial salpingectomy: a case report. Journal of Reproductive Medicine 1994; 39: 131-3.

4. Altinkaya SO, Ozat M, Pektas MK, Gungor T, Mollamahmutoglu L. Simultaneous bilateral tubal pregnancy after in vitro fertilization and embryo transfer. Taiwan Journal of Obstetrics and Gynaecology 2008; 47: 338-40.

5. Condous G, Okaro E, Khalid A. Prediction of ectopic pregnancy with the hCG ratio: a new biochemical marker. Human Reproduction 2003; 18(Suppl 1): 95-6.

6. Condous G, Okaro E, Khalid A, Lu C, Van Huffel S, Bourne T. The accuracy of transvaginal ultrasonography for the diagnosis of ectopic pregnancy prior to surgery. Human Reproduction 2005; 20: 1404-9. 